\title{
Une revue en évolution
}

\section{Lucie Sauvé et Étienne van Steenberghe}

\section{(2) OpenEdition}

Journals

Édition électronique

URL : http://journals.openedition.org/ere/944

DOI : $10.4000 /$ ere.944

ISSN : 2561-2271

\section{Éditeur}

Centr'ERE

\section{Édition imprimée}

Date de publication : 20 décembre 2016

ISSN : 1373-9689

\section{Référence électronique}

Lucie Sauvé et Étienne van Steenberghe, «Une revue en évolution », Éducation relative à l'environnement [En ligne], Volume 13 - 2 | 2016, mis en ligne le 20 décembre 2016, consulté le 24 septembre 2020. URL : http://journals.openedition.org/ere/944 ; DOI : https://doi.org/10.4000/ere.944 


\title{
Une revue en évolution
}

\author{
Lucie Sauvé et Étienne van Steenberghe
}

1 Cofondée en 1998 par Lucie Sauvé (Université du Québec à Montréal) et Louis Goffin (Fondation Universitaire Luxembourgeoise - Université de Liège, Belgique), la revue Éducation relative à l'environnement : Regards - Recherches - Réflexions s'appuie sur un partenariat avec différents groupes et instituts de recherche, en particulier l'Ifrée (Institut de formation et de recherche en éducation à l'environnement, France). Elle a comme point d'ancrage permanent une équipe de chercheurs de l'Université du Québec à Montréal, aujourd'hui regroupés au sein du Centre de recherche en éducation et formation relatives à l'environnement et à l'écocitoyenneté - Centr'ERE.

Cette revue est le seul périodique de recherche consacré à l'éducation relative à l'environnement au sein de la Francophonie - alors qu'on peut en dénombrer plus d'une dizaine dans d'autres langues. Il nous est apparu en effet que le rapport à l'environnement est indissociable de sa dimension culturelle, ce qui implique la reconnaissance et la valorisation de la spécificité des modes de rapport au monde et d'expression de celui-ci en Francophonie, perçue comme une sphère culturelle diversifiée. La revue permet donc de discuter, de partager et de diffuser les travaux de recherche produits ou exprimés ou traduits en langue française, favorisant ainsi une transculturalité féconde pour la recherche.

La revue se veut également interdisciplinaire, accueillant une diversité de domaines de savoirs et stimulant les différents croisements entre ceux-ci, de façon à contribuer au développement du vaste champ de l'éducation relative à l'environnement : certes, la pédagogie, la didactique et autres sciences de l'éducation y trouvent une place de choix, mais il importe d'inclure également les apports de la philosophie, de l'anthropologie, de la sociologie, de la géographie, des sciences de la nature, de la psychologie, de la muséologie, de l'art, etc. La revue valorise le dialogue des savoirs de divers types. Elle favorise le «métissage » théorique, méthodologique, culturel et autres.

4 La revue Éducation relative à l'environnement : Regards - Recherches - Réflexions s'intéresse ainsi à la diversité des fondements, des approches, des pratiques et des contextes - en somme à la pédagodiversité - qu'il importe de déployer pour favoriser un rapport plus harmonieux à l'environnement et une réflexion critique plus affinée sur les 
dynamiques éducatives à cet effet. Elle accueille à cet effet des productions de recherche du domaine spécifique de l'éducation ou de la formation relatives à l'environnement, mais aussi de champs associés comme ceux de l'éducation scientifique, l'éducation à la consommation, l'éducation au développement durable, l'éducation aux droits humains, l'éducation à la paix, etc., qui permettent de mieux appréhender le rapport personnel et social à l'environnement.

Par ailleurs, au-delà de sa mission de production et de diffusion de la recherche, la revue offre à travers sa dynamique d'évaluation des articles, une plateforme de coformation au sein de la communauté des chercheurs et des praticiens réflexifs. Elle devient un tremplin de publication pour les nouveaux chercheurs, qui y sont étroitement accompagnés.

Enfin, la revue Éducation relative à l'environnement : Regards - Recherches - Réflexions vise à favoriser l'arrimage étroit entre la recherche et l'intervention dans un processus d'enrichissement mutuel de la théorie et de la pratique. Elle contribue à structurer un réseau de chercheurs préoccupés de promouvoir, à travers les dynamiques éducatives, la qualité de notre rapport au monde. Elle invite les praticiens à porter un regard réflexif sur leurs actions éducatives et à diffuser et discuter leurs expériences. Elle s'adresse ainsi tant aux chercheurs et aux étudiants, qu'aux enseignants, aux formateurs, aux animateurs et autres éducateurs.

$7 \mathrm{Au}$ cours des 20 années de son existence, la revue aura ainsi permis de stimuler et de valoriser les travaux des chercheurs en éducation relative à l'environnement et autres champs associés. La production des volumes thématique a été associée à la tenue de colloques ou de séminaires permettant de partager et de discuter les recherches avant d'engager les travaux de publication. C'est ainsi qu'en 2019, seize numéros thématiques auront été produits.

-Vol. 1 : Les axes et pratiques de recherche

-Vol. 2 : Les enjeux de l'évaluation

-Vol. 3 : Le partenariat : pertinence, possibilités et enjeux

-Vol. 4 : Environnements, cultures et développements

- Vol. 5 : Cultures et territoires : des ancrages

- Vol. 6 : L'institution scolaire : réalités et enjeux politiques, stratégiques, pédagogiques

-Vol. 7 : La dimension critique de l'éducation relative à l'environnement

- Vol. 8 : La dimension éthique

- Vol. 9 : La dimension politique

- Vol. 10 : Le support de l'ancrage territorial

-Vol. 11 : Rapport au savoir, environnement et développement durable

-Vol. 12 : Questions d'identité et d'engagement

- Vol. 13 : La recherche associative

-Vol. 14 (2018) : L'art : un creuset du rapport à l'environnement

-Vol. 15 (2018) : Les aires protégées et les musées

-Vol. 16 (2019) : L'éducation relative à l'environnement auprès des adultes

Jusqu'à ce numéro ouvert (Vol 13-2), la revue était ainsi publiée environ une fois par an, autour d'une thématique spécifique. Pour mieux répondre au déploiement de la recherche contemporaine en éducation relative à l'environnement, deux numéros par an sont désormais publiés : un numéro thématique et un numéro ouvert à diverses propositions de publication. Cette stratégie permettra de mieux mettre en lumière le foisonnement des objets et des démarches de recherche dans ce domaine. 
9 Signalons que la revue est diffusée intégralement sous forme numérique, et ce dès les premiers numéros. Elle est également disponible en format imprimé. Et si on peut la consulter ou l'emprunter dans un nombre grandissant de bibliothèques particulièrement au sein de la Francophonie -, elle se trouve également en libre accès

10 Tout comme Geneviève Fioraso (2013), l'équipe éditoriale de la revue soutient en effet l'idée que les travaux de recherche devraient être accessibles à tous et pas seulement à ceux qui travaillent en milieu universitaire. C'est ainsi que pour une plus large diffusion, la revue a migré récemment sur le portail Revues.org et elle sera bientôt accessible sur la plateforme Érudit. Toutefois, la possibilité d'obtenir une version imprimée des numéros thématiques est toujours maintenue.

11 La production d'une telle revue est certes exigeante, en particulier dans un contexte de sous-financement de telles publications. L'importance du temps de travail requis a été mise en évidence dans plusieurs études (Solomon, D. J. et Björk B-C 2012 ; Contat, O. et Gremillet, A-S., 2015). Par ailleurs, il importe de contrer les publications « à rabais » de rigueur (Beall, J. ,2012), répondant à un contexte où, dans nos sociétés néolibérales, la course aux publications est devenue un enjeu majeur dans la vie professionnelle de nombreux universitaires. En réaction, Willinsky (2014) encourage les chercheurs à une plus grande responsabilité de leur statut public en publiant leurs travaux de recherches dans des revues rigoureuses qui donnent libre accès à leur contenu.

12 En ce sens, en raison de la rigueur de son processus de production et de la qualité des articles publiés, la revue Éducation relative à l'environnement : Regards - Recherches Réflexions est considérée comme une revue internationale exigeante. Depuis 2010, elle est classée Catégorie 1 par le Haut Conseil de l'évaluation de la recherche et de l'enseignement supérieur (HCERES, France).

13 Merci à tous les collaborateurs et collaboratrices, à tous les auteurs et auteures qui accordé leur confiance à la revue et y ont contribué. À titre d'exemples de contributions à la réflexion collective, voici diverses citations glanées dans chacun des volumes publiés jusqu'ici :

Les enjeux de l'éducation relative à l'environnement, liés à la viabilité des sociétés humaines, nous semblent trop importants pour laisser ce type d'éducation thématique baigner dans un contexte d'ambiguïté conceptuelle, d'improvisation des contenus et d'activisme didactique. Comme toute discipline scientifique, elle doit être fondée sur une véritable recherche. (Louis Goffin, vol. 1, p. 48)

On voit dans l'évaluation un puissant moyen de réduction de ses erreurs et, surtout, on se dit que l'autre, l'apprenant, a quelque chose à nous dire sur nos méthodes, nos comportements, nos choix d'éducateur. Évaluer c'est aussi écouter, être attentif à la personne insérée dans le système formatif que l'on a établi pour elle (...). Mais qui prétend à l'objectivité quand il est impliqué dans le processus pédagogique évalué, celui-là n'est pas humain. La subjectivité est là, et mieux vaut l'éclairer et l'intégrer à l'analyse comme facteur influant plutôt que de la réfuter (...) Je sais que mon propre rapport sensible à l'environnement maritime agit secrètement sur le processus. (Dominique Cottereau, vol. 2, p. 174,179)

L'éducation relative à l'environnement s'est fondée à la rencontre d'une critique de la société, de ses modes de production et de consommation, et d'une remise en cause de l'école et de son fonctionnement; elle visait à modifier, par l'éducation, le rapport des individus à la société et au monde. (Jean-Étienne Bidou - vol. 3, p. 10)

Nos environnements, ceux des villes, des milieux agricoles, des régions montagneuses, des zones littorales, etc., se construisent sur une trame à double dimension, celles de la nature et de la culture (qui exprime l'humain dans la nature). À la fois, ils déterminent notre rapport au monde comme ils témoignent de 
ce dernier. Ils induisent comme ils traduisent les caractères fondamentaux de nos rapports sociaux, de nos " choix de société ", de nos " modes de développement ". (Lucie Sauvé et Renée Brunelle - Vo. 4, p. 8)

Le territoire n'existe pas en soi. Il n'a de réalité qu'à travers celui qui s'y trouve. Il correspond le plus souvent à un espace physiquement délimité, aménagé et au cœur d'une histoire collective ou individuelle. Il s'agit donc d'un lieu socialisé où l'identité culturelle et l'identité géographique se fondent dans un même espace. (Étienne van Steenberghe - vol. 5, p. 122)

L'éducation relative à l'environnement porte sur le rapport au monde, la sphère de la relation au milieu de vie, l'écoformation. Ni science biophysique, ni science humaine, elle est avant tout pour moi à définir par son objet, notre rapport au monde. Elle ne peut ainsi se satisfaire d'une focalisation sur les problèmes environnementaux. (Tom Berryman - vol. 6, p. 142)

L'éducation relative à l'environnement, dans ses courants les plus critiques, est consciente des dommages infligés à la Terre et à l'humanité et elle reconnaît dans l'appel au réenchantement du monde, non pas un retour au passé, selon une conception réactionnaire et passéiste de l'aventure humaine, mais une exigence, celle de faire advenir un nouvel espace-temps historique, une nouvelle configuration sociale concrète qui mixerait écologie et lien social, justice environnementale et justice économique. (Mohamed Taleb - vol. 8, p. 83)

Développer une vision « Pour Nous Tous » d'un territoire donné ne se fait pas du jour au lendemain et implique un travail de socialisation en profondeur, mais aussi d'émancipation des uns à l'égard des autres et d'écocipation (appropriation de son environnement) : le cadre environnemental est non seulement pris en compte, mais le but, c'est qu'il devienne pour les participants un véritable territoire d'action. La finalité est donc que les participants s'impliquent, s'engagent par rapport à leurs espaces publics en particulier, et plus largement par rapport à leur environnement. (Christine Partoune, vol. 10, p. 120)

L'éducation contemporaine est interpellée au premier plan pour accompagner et stimuler le développement d'une écocitoyenneté critique, créative et engagée, capable et désireuse de participer aux débats publics, à la recherche de solutions et à l'avènement d'une écosociété. À cet effet, parmi les apprentissages désormais nécessaires, tant en milieu scolaire que tout au long de la vie, il faut souligner les compétences d'ordre critique, éthique et politique. Ces champs de compétences intègrent un ensemble de savoirs de divers types et ils sont étroitement liés entre eux. Ils font appel à une " pédagogie engagée » et soulèvent des défis stimulants pour les acteurs d'une éducation ancrée, en toute pertinence, dans un monde en mutation. (Lucie Sauvé - vol. 11, p. 19)

Le dialogue des savoirs, intégrant une perspective critique, émerge et se nourrit dans la construction des luttes, des rêves et des espoirs, ce qui implique la connaissance et la reconnaissance des multiples dimensions de l'être humain. Tel que Morin le signale, cela crée les conditions pour que chacun se « situe » dans le (son) monde, dans la (sa) terre, dans l'(son) histoire et dans la (sa) société, apprenant à problématiser, à s'interroger et à interroger, à contextualiser. (Isabel Orellana et Marie-Ève Marleau - vol. 12, p. 70)

Il ne suffit pas de convier praticiens et chercheurs à mêmes évènements ou à les rassembler dans des projets de recherche communs. Il faut aussi construire les méthodes. C'est sur ce point que les difficultés sont particulièrement nombreuses et les résistances individuelles et institutionnelles fortes. Mais plus encore, le clivage extrêmement profond qui sépare à la fois, dans leur nature et aussi dans les pratiques, savoirs savants et savoirs profanes, (Jean-Étienne Bidou - vol. 13, p. 10) mettent en évidence une diversité d'aspects et d'enjeux en éducation relative à l'environnement, de même qu'une diversité de méthodologies de recherche. Ainsi sont abordés les objets de recherche suivants : l'éducation relative à la citoyenneté chez les 
enfants, l'éthique professionnelle des enseignants au regard de l'éthique de la relation à la nature, les ancrages épistémologiques de l'éducation au territoire, la dimension sociale de l'éducation au changement climatique, les enjeux socio-politiques dans la mise en œuvre de l'apprentissage par problèmes (APP), l'analyse d'une démarche de résolution de problèmes environnementaux en République de Guinée, les dimensions phénoménologique et praxique de l'éducation à l'alimentation, l'éducation relative à la consommation au primaire selon l'approche de philosophie pour enfants, les possibilités et limites de l'éducation au développement durable au Chili.

Ainsi le champ de l'éducation relative à l'environnement ne cesse de se déployer en diversité, dans différents contextes, répondant à une pluralité de situations ou de préoccupations socio-écologiques persistantes, mouvantes ou émergeantes.

Bonne lecture!

\section{BIBLIOGRAPHIE}

Beall, J. (2012). Predatory publishers are corrupting open access. Consulté le 10 novembre 2016 sur « nature.com » : https://www.nature.com/news/predatory-publishers-are-corrupting-openaccess- 1.11385

Contat, O. et Gremillet, A-S. (2015). Publier : à quel prix ? Étude sur la structuration des coûts de publication pour les revues françaises en SHS. Revue française des sciences de l'information et de la communication, 7 [En ligne]. Consulté le 2 novembre 2016 : http://rfsic.revues.org/1716

Fioraso, G. (2013). L'information scientifique est un bien commun qui doit être disponible pour tous. Discours lors des 5e journées Open Access, le 24 janvier 2013 [En ligne]. Consulté le 27 novembre 2016 : http://www.enseignementsup-recherche.gouv.fr/cid66992/discours-degenevieve-fioraso-lors-des-5ejournees-open-access.html

Solomon, D. J. et Björk B-C (2012). A Study of Open Access Journals Using Article Processing Charges. Journal of the American Society for Information Science and Technology, $n^{\circ} 63, \mathrm{p} .1485-$ 1495.

Willinsky, J. (2014). The new openness in educational research. Dans A. Reid, P. Hart et M. Peters (eds.), A companion to research in education (p. 575-582). New York, NY

\section{INDEX}

Keywords : environmental education, learned journals

Mots-clés : revue savante, éducation relative à l'environnement 
AUTEURS

LUCIE SAUVÉ

Directrice de la revue

ÉTIENNE VAN STEENBERGHE

Rédacteur en chef 